\title{
Automatic Ambulance System using IOT
}

\author{
Himadri Nath Saha ${ }^{1}$, Arpita Konar², Shreya Guha², Avik Mukherjee ${ }^{2}$, Shayeli \\ Sarker $^{2}$, Saunak Chowdhury², Saanya Khan ${ }^{2}$, Gourav Saha², Rituparna \\ Guchhait $^{2}$, Aniket Mittra ${ }^{2}$, Sanhita Bishal ${ }^{2}$, Srutayu Biswas ${ }^{3}$ \\ ${ }^{1}$ Surendranath Evening College, Calcutta University, Kolkata, India \\ ${ }^{2}$ Institute of Engineering And Management Kolkata, India \\ ${ }^{3}$ Heritage Institute of Technology, Kolkata, India
}

\begin{abstract}
With the Internet of Things (IoT) gradually evolving as the subsequent phase of the evolution of the Internet, it becomes crucial to recognize the various potential domains for application of IoT, and the research challenges that are associated with these applications. Ranging from smart cities, to health care, smart agriculture, logistics and retail, to even smart living and smart environments IoT is expected to infiltrate into virtually all aspects of daily life. Even though the current IoT enabling technologies have greatly improved in the recent years, there are still numerous problems that require attention. Since the IoT concept ensues from heterogeneous technologies, many research challenges are bound to arise. The fact that IoT is so expansive and affects practically all areas of our lives, makes it a significant research topic for studies in various related fields such as information technology and computer science. Thus, IoT is paving the way for new dimensions of research to be carried out. This paper presents the recent development of IoT technologies and discusses future applications and research challenges on Mobile Health Hub as well.
\end{abstract}

KEYWORDS:IoT,IoNT,IoE, ICN, Science, Gateway, ,DataFrameworks,DataScience,Microservices

\section{INTRODUCTION}

Internet of Things (IoT) term represents a general concept for the ability of network devices to sense and collect data from around the world, and then share that data across the Internet where it can be processed and utilized for various interesting purposes. The IoT consists of smart machines interacting and communicating with other machines, objects, environments and infrastructures. Nowadays every person is connected with each other using lots of communication ways. The primary goal of this research paper is in accessing the patient's health status is to measure the patient's vitals which include heartbeat rate, blood pressure, body temperature etc. Sensors are available to measure almost every vital sign of a patient. These sensors can be used in the emergency vehicle to estimate the health condition of the patient. Apart from the sensor readings a camera module is also used this helps to capture the video of the patient. Since this information should reach the hospital prior to his arrival, a proper high speed communication methodology is required.

\section{ARCHITECTURE OF THE PROPOSED MODEL}

2.1. Ambulance module:

This model is to be used inside the ambulance The architecture consists of the Raspberry Pi with the sensor units interface to it. The patient's body parameters are measured using sensors. The parameters include heartbeat, temperature and blood pressure, blood glucose, cholesterol levels, and many others. This unit also includes a camera module to capture the image of the patient at regular intervals.

After processing the ambulance module upload the data to the cloud.

2.2. Cloud or Wifi module : 
The Cloud module consists of two cloud services. They are ThinkSpeak and Dropbox. The ThinkSpeak cloud platform supports numerical data to be uploaded. The ThinkSpeak cloud also supports graphical representation of the uploaded data. The image uploading is done on Dropbox cloud.

\subsection{Hospital module}

In the hospital module the doctor monitors the data uploaded into the cloud. The red color detected image helps the doctors to identify the severity of the wounds. The hospital module consists of a simple application that can download the data from the cloud. With data received the doctors prepare for immediate medical response.

\section{APPLICATIONS}

Application domains in this sector include being able to monitor a patient's compliance with prescriptions, telemedicine solutions, and alerts for patients' well-being. Thereby, sensor devices that can be automated and enhanced through technology. Additional technology that can facilitate various operations like report sharing to multiple individuals and locations, record keeping and dispensing medications would go a long way in changing the healthcare sector. A lot of benefits that IoT application offers in the healthcare sector is most categorized into tracking of patients, staff, and objects, identifying, as well as authenticating, individuals, and the automatic gathering of data and sensing. Hospital workflow can be significantly improved once patient flow is tracked. Additionally, authentication and identification reduce incidents that may be harmful to patients, record maintenance and fewer cases of mismatching infants. In addition, automatic data collection and transmission is vital in process automation, reduction of form processing timelines, automated procedure auditing as well as medical inventory management. Sensor devices allow functions centered on patients, particularly, in diagnosing conditions and availing real-time information about patients' health indicators Application domains in this sector include; being able to monitor a patient's compliance with prescriptions, telemedicine solutions, and alerts for patients' well-being. Thereby, sensors can be applied to outpatient and inpatient patients, dental Bluetooth devices and toothbrushes that can give information after they are used and patient's surveillance. Other elements of IoT in this capacity include; RFID, Bluetooth, and Wi-Fi among others. These will greatly enhance measurement and monitoring techniques of critical functions like blood pressure, temperature, heart rate, blood glucose, cholesterol levels, and many others. The applications of Internet of Things (IoT) and Internet of Everything (IoE) are further being extended through the materialization of the Internet of Nano-things (IoNT)

\section{METHODOLOGY}

The patient's health condition can be identified using Image processing. Image processing can be used to identify the extent of injury by detecting the amount of blood spread due to injury. But the drawback of this application is that it is specific to accident cases where there should be open wounds on the patient's body. This method is not applicable for emergency cases like cardiac arrest; stroke etc. where open wounds are missing. The existing emergency vehicle system video surveillance feature can be used as an add-on which helps the doctor to have a close look on the patient even before the patient reaches the hospital. Numerous sensors help in

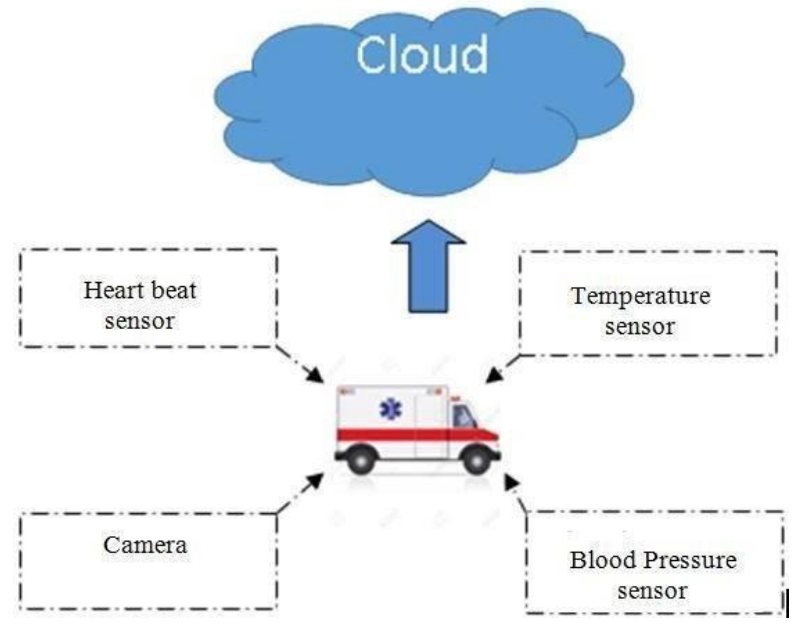


precisely estimating the health condition of the patient. These sensors can be used to measure blood pressure, heart beat rate, temperature etc. These collected data help the doctor to determine the health condition of the patient. If these real-time data can be made available for the access to the doctor, it can help him to give adequate feedback to the emergency vehicle as well as arrange hospital facilities for the patient.

- All Detection: Assistance for elderly or disabled people living independent.

- Medical Fridges: Monitoring and Control of conditions inside freezers storing medicines, vaccines, and organic elements.

- Sportsmen Care: Vital signs monitoring in high performance centers and fields.

- Patients Surveillance: Monitoring of conditions of patients inside hospitals and in old people's homes.

- Ultraviolet Radiation: Measurement of UV sun rays to warn people not to be exposed in certain hours.

\section{EXPERIMENTAL SETUP:}

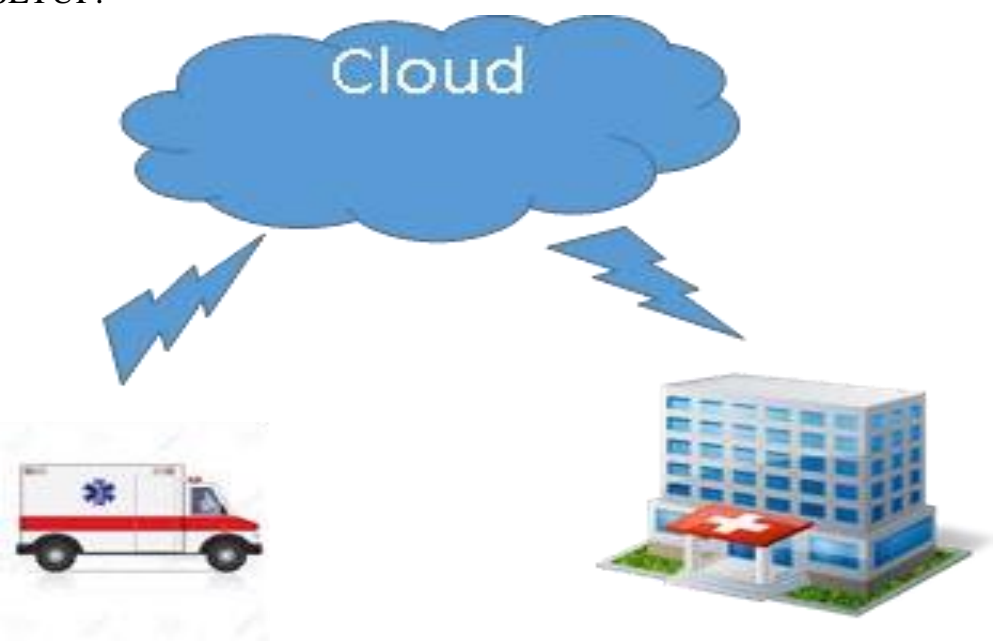

It will include all the modules as mentioned.Along with that the DHT11 Sensor would be used.For measuring the body temperature,we are using a DHT11 temperature sensor. It is a thermistor mounted on the surface. The temperature measured is transmitted serially to the controller. For measuring heart beat we are using NSK TCRT1000 heart rate sensor. It works based on the principle of photoplethysmography . For measurement purposes, a light detector and a light source are placed on the same side inside the sensor. Then the finger is placed on the opposite side. Thereafter the light source emits light which is passed into the finger of the patient and the light reflected is measured by the light detector placed inside the sensor. The amount of light reflected by the finger varies in accordance with the blood flow caused due to the heart beating. The system provides a channel of communication between ambulance and hospital modules. This can be employed in other applications. The system can be modified to act as a remote healthcare unit where the doctor can monitor patients remotely.

\section{HEALTHCARE}

A lot of benefits that IoT application offers in the healthcare sector is most categorized into tracking of patients, staff, and objects, identifying, as well as authenticating, individuals, and the automatic gathering of data and sensing. Hospital workflow can be significantly improved once patient flow is tracked. Additionally, authentication and identification reduce incidents that may be harmful to patients, record maintenance and fewer cases of mismatching infants. In addition, automatic data collection and transmission is vital in process automation, reduction of form processing timelines, automated procedure auditing as well as medical inventory management. Sensor devices allow functions centered on patients, particularly, in diagnosing conditions and availing real-time information about patients' health indicators.Application domains in this sector include; being able to monitor a patient's compliance with prescriptions, telemedicine solutions, and alerts for patients' well-being.

\section{EXTENDED CONCEPTS TO BE USED:}

The applications of Internet of Things (IoT) and Internet of Everything (IoE) are further being extended through the materialization of the Internet of Nano-things (IoNT). The notion of IoNT, as the name implies, is 
being engineered by integrating Nano-sensors in diverse objects (things) using Nano networks. Medical application is one of the major focuses of IoNT implementations. Application of IoNT in the human body, for treatment purposes, facilitates access to data from in situ parts of the body which were hitherto in accessible to sense from or by using those medical instruments incorporated with bulky sensor size. Thus, IoNT will enable new medical data to be collected, leading to new discoveries and better diagnostics.

\section{RESEARCH CHALLENGES:}

For all the above potential applications of IoT, there has to be proper feasibility into the different domains to ascertain the success of some applications and their functionality. As with any other form of technology or innovation, IoT has its challenges and implications that must be sorted out to enable mass adoption. Even though the current IoT enabling technologies have greatly improved in the recent years, there are still numerous problems that require attention, hence paving the way for new dimensions of research to be carried out. Since the IoT concept ensues from heterogeneous. Another research direction as regards data management is applying Information Centric Networking (ICN) in the IoT. Since these information centric systems offer support in the efficient content retrieval and access to services, they appear to be quite valuable not just in accessing but also transferring as well as managing generated content and its transmission. This solution, however, brings about various challenges such as; how to extend the ICN paradigm competently over the fixed network edge, how to take in IoTs static and mobile devices as well as how to apportion the functionality of ICN on resource constrained devices

\section{CONCLUSION:}

The IoT promises to deliver a step change in individuals" quality of life and enterprises" productivity. Through a widely distributed, locally intelligent network of smart devices, the IoT has the potential to enable extensions and enhancements to fundamental services in transportation, logistics, security, utilities, education, healthcare and other areas, while providing a new ecosystem for application development. This will be beneficial in health insurance,diagnosis of health problems at the earliest.

\section{REFERENCES:}

[1]. H. N. Saha, N. F. Raun and M. Saha, "Monitoring patient's health with smart ambulance systems using Internet of Things (IOTs)," 2017 8th Annual Industrial Automation and Electromechanical Engineering Conference (IEMECON), Bangkok, 2017, pp. 91-95.

[2]. Advanced IOT based combined remote health monitoring, home automation and alarm systemJ Saha, AK Saha, A Chatterjee, S Agrawal, A Saha, A Kar, HN Saha 2018 IEEE 8th annual computing and communication workshop and conference ...

[3]. Health monitoring using internet of things (iot) HN Saha, S Auddy, S Pal, S Kumar, S Pandey, R Singh, AK Singh, ...2017 8th Annual Industrial Automation and Electromechanical Engineering ...

[4]. Recent trends in the Internet of Things HN Saha, A Mandal, A Sinha 2017 IEEE 7th annual computing and communication workshop and conference ...

[5]. E. Borgia, D. G. Gomes, B. Lagesse, R. Lea, and D. Puccinelli, "Special issue on" Internet of Things: Research challenges and Solutions".," Computer Communications, vol. 89, no. 90, pp. 1-4, 2016.

[6]. Internet of Things (IOT): Research Challenges and Future Applications AbdelRahman H. Hussein Department of Networks and Information Security Faculty of Information Technology / Al-Ahliyya Amman University

[7]. IoT based mobile health hub :Diljo Thomas, Vineeth V L, Siddharth P G and Shanmugasundaram M School of Electronics Engineering, VIT University, Vellore 632 014, Tamil Nadu, India

[8]. Anang Hudaya Muhamad Amin, Nazrul Muhaimin Ahmad, Afiq Muzakkir Mat Ali 2016 Decentralized Face Recognition Scheme for Distributed Video Surveillance in IoT-Cloud Infrastructure 2016 IEEE Region 10 Symposium (TENSYMP), Bali, Indonesia

[9]. Hoda Ramin Hossein M S, and Shaikh S S 2016 SPHPMS: Smart Personnel m-Healthcare Patient Monitoring System International Conference on Electrical, Electronics, and Optimization Techniques (ICEEOT).

[10]. Andrew Yearp, David Newell, Philip Davies, Russell Wade and Reza Sahandi 2016 Wireless Remote Patient Monitoring System: Effects of Interference 2016 10th International Conference on Innovative Mobile and Internet Services in Ubiquitous Computing.

[11]. Mithun Chandra Paul, Suman Sarkar, Mahfujur Rahman MD and Sayed Mohsin Reza 2016 Low Cost and Portable Patient Monitoring System for e-Health Services in Bangladesh 2016 International Conference on Computer Communication and Informatics (ICCCI 2016). 1234567890 14th ICSET-2017 IOP Publishing IOP Conf. Series: Materials Science and Engineering 263 (2017) 052048 doi:10.1088/1757-899X/263/5/052048

[12]. Sakshi Sharma and Rashmi Vashisht 2015 Zigbee Based Centralized Patient Monitoring System International Conference on Computational Intelligence and Communication Networks.

[13]. Arun Fera M, R.Ashwin, Santhiya M, Gayathiri Deepa K.R.and Thangaprabha M 2015 HEAL \pm Health monitoring in Emergency vehicles with their Authentication by RFID and Location tracking by GPS 2015 Seventh International Conference on Advanced Computing. 